\title{
A General Model for Hepatitis B Disease with Age-Dependent Susceptibility and Transmission Probabilities
}

\author{
Danga Duplex Elvis Houpa, Tagne Eric Miamdjo, Tchaptchie Yannick Kouakep* \\ Department of Mathematics and Computer Science, Ngaoundéré University, Ngaoundere, Cameroon \\ Email: e houpa@yahoo.com, ericmiamdjo5@gmail.com, "kouakep@aims-senegal.org
}

Received 9 December 2013; revised 9 January 2014; accepted 17 January 2014

Copyright $@ 2014$ by authors and Scientific Research Publishing Inc.

This work is licensed under the Creative Commons Attribution International License (CC BY).

http://creativecommons.org/licenses/by/4.0/

(c) (i) Open Access

\begin{abstract}
A SEI model for hepatitis B is constructed where the susceptibility and other crucial transmission probabilities depend on the chronological age and the basic reproduction rate $R_{0}$ is derived. Under suitable (biological and mathematical) assumptions in a closed population, results of Houpa D. D. E. et al. [1] are extended from constant case of $p$ and $q$ to age-dependent case: the disease-free equilibrium is globally asymptotically stable (GAS) if $R_{0}<1$. On the other hand, $R_{0}>1$ induces that endemic equilibrium is GAS and the system is uniformly persistent.
\end{abstract}

Keywords

Hepatitis B; PDE Model; Global Stability; Lyapunov-LaSalle Functionals

\section{Introduction}

This paper studies a system of equations modelling the dynamic of hepatitis B with age-dependent susceptibility in a closed population. Its manifestations in human body are shown by hepatitis B antigens (small spherical particles, tubular forms and a large shelled spherical particles) because of their association with a high risk of hepatitis [2]. Hepatitis B caused acute hepatitis and severe chronic liver disease. Hepatitis is endemic in Africa [3] [4]. According to Pasquini et al. [5] (with a computer model), Bonzi et al. [6] (with an EDOs model), Inaba et al. [7] (theoretically with a PDE) or D. J. Nokes et al. [8] (with statistics tools) and L. Zou et al. [9] (with PDE by fitting model to data), age factor is important in epidemiology of disease like hepatitis and reveals most of time useful informations on the dynamics of the epidemic.

${ }^{*}$ Corresponding author.

How to cite this paper: Houpa, D.D.E., Miamdjo, T.E. and Kouakep, T.Y. (2014) A General Model for Hepatitis B Disease with Age-Dependent Susceptibility and Transmission Probabilities. Applied Mathematics, 5, 707-722.

http://dx.doi.org/10.4236/am.2014.54068 
A SEI model for hepatitis B is constructed where the susceptibility and other crucial transmission probabilities depend on the chronological age and the basic reproduction rate $R_{0}$ is derived. Under suitable (biological and mathematical) assumptions in a closed population, it is proved that the disease-free equilibrium is globally asymptotically stable (GAS) if $R_{0}<1$, and $R_{0}>1$ induces that endemic equilibrium is GAS and the system is uniformly persistent.

The work is organized as follows. After the presentation of the mathematical model with its main results, Section 2 studies the well posedness of the PDE and derives preliminary results useful to study the long-term behaviour of the model. Moreover, it deals with the wellposedness of the model and proves the global asymptotic stability of the disease-free equilibrium when the basic reproduction number $R_{0}<1$ and stability of the endemic equilibrium (EE) with the carriers $(E)$ transmission rate $\beta_{E}$ small enough to be considered as zero. These results are verified through numerical simulations extended by a discussion and conclusions in Section 3 .

\section{Mathematical Model}

\subsection{Presentation}

In this study we will consider the following (chronological) age-dependent susceptibility model:

$$
\begin{aligned}
& \left(\partial_{t}+\partial_{a}\right) S(t, a)=-m(a) S(t, a)-\lambda(t, a) S(t, a), a>0 \\
& S(t, 0)=\Lambda, t>0 \\
& \frac{\mathrm{d} E(t)}{\mathrm{d} t}=\int_{0}^{+\infty} p(a) \lambda(t, a) S(t, a) \mathrm{d} a-\mu_{E} E(t) \\
& \frac{\mathrm{d} I(t)}{\mathrm{d} t}=\int_{0}^{+\infty} q(a) \lambda(t, a) S(t, a) \mathrm{d} a-\mu_{I} I(t)+\varepsilon E(t)
\end{aligned}
$$

posed for $t>0$ and $a>0$. Here $s(t, a)$ denotes the age-specific density of susceptible, $E(t)$ and $I(t)$ denotes respectively the age-specific densities of acute infected (that can be symptomatic or asymptomatic) and chronic carriers. In addition $p \in L_{+}^{\infty}(0, \infty)$ is a given function such that $0<p(a) \leq 1$ a.e. while $q(a) \equiv 1-p(a)$. Function $q$ represents the age-specific probability to become a chronic carrier when becoming infected. Function $p$ denotes the probability to develop an acute infection when getting the infection age $a$ ([8] studied the age-dependence susceptibility to the infection). We conditionally extend in some sens results of Houpa D. D. E. et al. [1] who analyzed the case where $p$ and $q$ are constant. Parameter $m(a)>0$ denotes the natural death rate at age $a, \mu_{I}>0$ and $\mu_{E}$ denotes the exit rates associated to each infected class. Clearly at each age $a>0,0 \leq m(a) \leq \min \left(\mu_{I}, \mu_{E}\right) . \varepsilon>0$ is the transition rate from $E$ to $I$. Obviously, $\mu_{E} \geq \varepsilon$. In some studies (like Kouakep et al. [10]) authors set $\mu_{E} \geq \mu_{I}$. The term $\lambda(t, a)$ corresponds to the age-specific force of infection and follows the usual law of mass-action, that reads as

$$
\lambda(t, a)=\beta_{I}(a) I(t)+\beta_{E}(a) E(t) .
$$

This problem (1.1) is supplemented together with the boundary conditions:

$$
\begin{aligned}
& S(t, 0)=\Lambda, \text { constant influx) } \\
& E(0)=E_{0}, \\
& I(0)=I_{0},
\end{aligned}
$$

and initial data

$$
S(0, a)=S_{0}(a) .
$$

This model (1.1) is suggested by Melnik et al. [11] for the age-dependent susceptibility concept supplemented with Kouakep et al. [10] introducing $p$ and $q$.

We recall that according to WHO [4], Bonzi et al. [6] and Fall et al. [3], asymptomatic carriers has a low infectious rate. As a consequence in most part of this work one will assume that

$$
0 \approx \beta_{E}(a) \ll \beta_{I}(a) .
$$

Then 


$$
\lambda(t, a)=\beta_{I}(a) I(t) .
$$

In the above model (1.1)-(1.5), we do not take into account possible vertical transmission and we do not consider any control strategy such as vaccination campaign. It seems to be relevant together the assumption of WHO [4] wich considers that vertical transmission of the disease exists in sub-Saharan Africa. But its influence on the dynamics of the disease is rather small because the proportion of chronic infections acquired perinatally is low [12].

Using the data, Nokes et al. in [8] constructed the prototype (useful for us) in the simulations:

$$
q(a)=\mathrm{e}^{-0.645 a^{0.455}}, a>0 .
$$

We do not focus on chronological age in the infective classes.

\subsection{Main Results and Simulations}

The basic reproduction rate is defined by

$$
R_{0}=\frac{1}{\mu_{I} \mu_{E}} \int_{0}^{+\infty}\left(\varepsilon p(a)+\mu_{E} q(a)\right) \beta_{I}(a) S_{F}(a) \mathrm{d} a
$$

The DFE is defined by

$$
\left(S_{F}(a), E_{F}, I_{F}\right)=\left(\Lambda \exp \left(-\int_{0}^{a} m(\sigma) \mathrm{d} \sigma\right) ; 0 ; 0\right) .
$$

For endemic equilibrium, we obtain only in the case $R_{0} \geq 1$,

$$
S_{e}(a)=\Lambda \exp \left(-\int_{0}^{a} m(\sigma)+I_{e} \beta_{I}(\sigma) \mathrm{d} \sigma\right) .
$$

linked to

$$
\frac{1}{\mu_{I} \mu_{E}} \int_{0}^{+\infty}\left(\varepsilon p(a)+\mu_{E} q(a)\right) \beta_{I}(a) S_{e}(a) \mathrm{d} a=1
$$

That means

$$
\frac{1}{\mu_{I} \mu_{E}} \int_{0}^{+\infty}\left(\varepsilon p(a)+\mu_{E} q(a)\right) \beta_{I}(a) S_{F}(a) \exp \left(-\int_{0}^{a} I_{e} \beta_{I}(\sigma) \mathrm{d} \sigma\right) \mathrm{d} a=1 .
$$

Assumption 1. Assume that the maps $a \mapsto \beta_{I}(a)$ is bounded and uniformly continuous from $[0, \infty)$ into itself.

Let $G(x)=x-\ln x-1$. The function $G$ has only one extremum which is a global minimum 0 at 1 , satisfying $G(1)=0$ (see [13]). We make these assumptions for the endemic equilibrium $\left(S_{e}(a), E_{e}, I_{e}\right)$ when $R_{0}>1$ :

Assumption 2.

1. $H(t, a)=S_{e}(a)\left(\frac{S(t, a)}{S_{e}(a)}-1\right)\left(\frac{\partial_{a} S(t, a)}{S(t, a)}+m(a)+\beta_{I}(a) I_{e}\right)$ has a constant sign on $[0 ;+\infty)^{2}$.

2. On the attractor $\mathcal{A}$ (an invariant compact attractor of all bounded sets following the Proposition 2 therein), the following inequality holds true:

$$
G\left(\frac{I(t) E_{e} S(t, a)}{I_{e} E(t) S_{e}(a)}\right)+G\left(\frac{I_{e} E(t)}{E_{e} I(t)}\right)-G\left(\frac{S(t, a)}{S_{e}(a)}\right) \geq 0 .
$$

We make also this assumption for the disease free equilibrium $\left(S_{F}(a), 0,0\right)$ when $R_{0}<1$ :

Assumption 3. $P(t, a)=\left(\frac{S(t, a)}{S_{F}(a)}-1\right)\left(\frac{\partial_{a} S(t, a)}{S(t, a)}+m(a)\right)$ has a constant sign on $[0 ;+\infty)^{2}$.

The global stability of the steady states is resumed in the following Theorem 1.

Theorem 1. Assume Assumptions 1, 2 and 3. Then: 
- If $R_{0}=\frac{1}{\mu_{I} \mu_{E}} \int_{0}^{+\infty}\left(\varepsilon p(a)+\mu_{E} q(a)\right) \beta_{I}(a) S_{F}(a) \mathrm{d} a<1$, then the DFE, the disease free equilibrium, is globally asymptotically stable.

- If $R_{0}>1$, then there exists an endemic equilibrium that is globally asymptotically stable for all $S>0$, $E>0$ and $I>0$. Moreover, in that case $\left(R_{0}>1\right)$ the system is uniformly persistent.

Remark 1. We will see that disease free equilibrium (DFE) exists whenever $R_{0}>1$ or $R_{0} \leq 1$. But endemic equilibrium exists only when $R_{0} \geq 1$.

We denote in Tables 1 and 2: "p" for people(s), “yr" for year and "nbb” for "new born babies". We made simulations with the values in Tables 1 and 2 and denoted by $\Lambda$ the constant birth rate at any positive time (with year unit). We consider the following parameters for DFE case $\left(R_{0}=10^{-5}<1\right.$ related to Figures 1-3).

For endemic case ( $R_{0}=2.20>1$ related to Figures 4-10), we consider the values in the Table 2 .

We have tested our Assumption 2-2 on the Figures 9 and 10 with $E_{e}=12.63$ and $I_{e}=405.83$ : it is verified in our simulations up to some time (considered as origin by time shifting or rescalling, wich is not very important in our case for long term dynamics in our simulations or calculations) with the global asymptotic stability of the endemic case $\left(R_{0}>1\right)$. One could see that Assumption 2 and 3 could be relaxed by proving them for $t \geq t_{0}$ with $t_{0}$ an arbitrary positive real constant (or number). In all the cases, we observe a period of stability after a severe outbreak of the disease.

\subsection{Technical Materials}

Let us introduce the Banach space $X=L^{1}(] 0 ;+\infty[, \mathbb{R}) \times \mathbb{R}^{3}$ and $\left.\left.X_{0}=L^{1}(] 0 ;+\infty\right), \mathbb{R}\right) \times\{0\} \times \mathbb{R}^{2}$ endowed with the usual product norm as well as its positive cone $X_{+}$defined (with $\mathbb{R}_{+}=[0 ;+\infty[$ ) by:

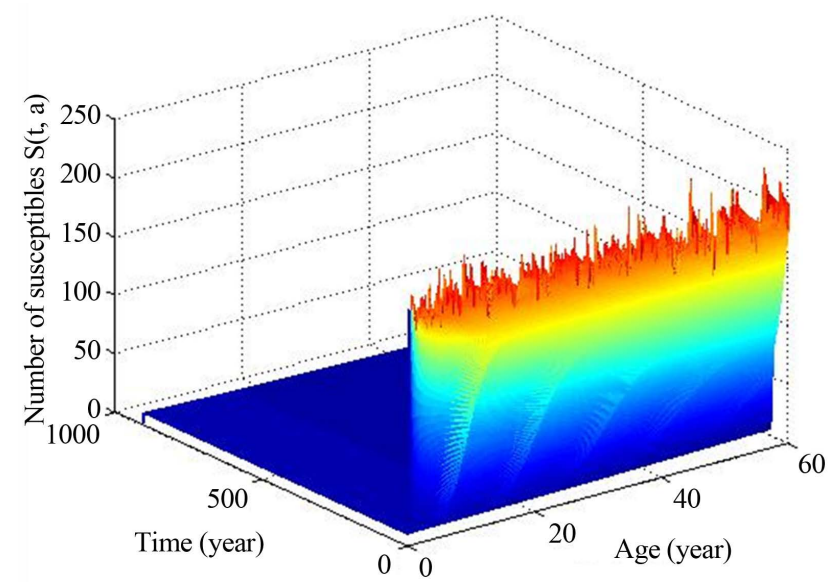

Figure 1. Function $S(t, a)$ with $R_{0}<1$.

Table 1. Values for $R_{0}<1$.

\begin{tabular}{ccccccccc}
\hline Parameters/function & Age & $q$ & $\beta_{I}$ & $\varepsilon$ & $\mu_{I}$ & $\mu_{E}$ & $m(a)$ & $\Lambda$ \\
\hline Value(s) & {$[0 ; \mathrm{A}=60]$} & $\mathrm{e}^{-0.6455^{0.455}}$ & 0.000000008 & 0.00001 & 0.2018458 & 8.1 & 0.18 & 10 \\
Unit & $\mathrm{yr}$ & probability & $(\mathbf{p} \cdot \mathrm{yr})^{-1}$ & $(\mathbf{p} \cdot \mathrm{yr})^{-1}$ & $\mathrm{yr}^{-1}$ & $\mathrm{yr}^{-1}$ & $\mathrm{yr}^{-1}$ & $\mathrm{Nbb} / \mathrm{yr}^{-1}$ \\
\hline
\end{tabular}

Table 2. Values for $R_{0}<1$.

\begin{tabular}{ccccccccccc}
\hline Parameters/function & Age & $q$ & $\beta_{I}$ & $\varepsilon$ & $\mu_{I}$ & $\mu_{E}$ & $m(a)$ & $\Lambda$ & 0.018 & 10 \\
Value(s) & {$[0 ; \mathrm{A}=60]$} & $\mathrm{e}^{-0.645 a^{0.455}}$ & 0.0001 & 0.1 & 0.02018458 & 0.1 \\
Unit & $\mathrm{yr}$ & probability & $(\mathbf{p} \cdot \mathrm{yr})^{-1}$ & $(\mathbf{p} \cdot \mathrm{yr})^{-1}$ & $\mathrm{yr}^{-1}$ & $\mathrm{yr}^{-1}$ & $\mathrm{yr}^{-1}$ & $\mathrm{nbb} / \mathrm{yr}^{-1}$ \\
\hline
\end{tabular}




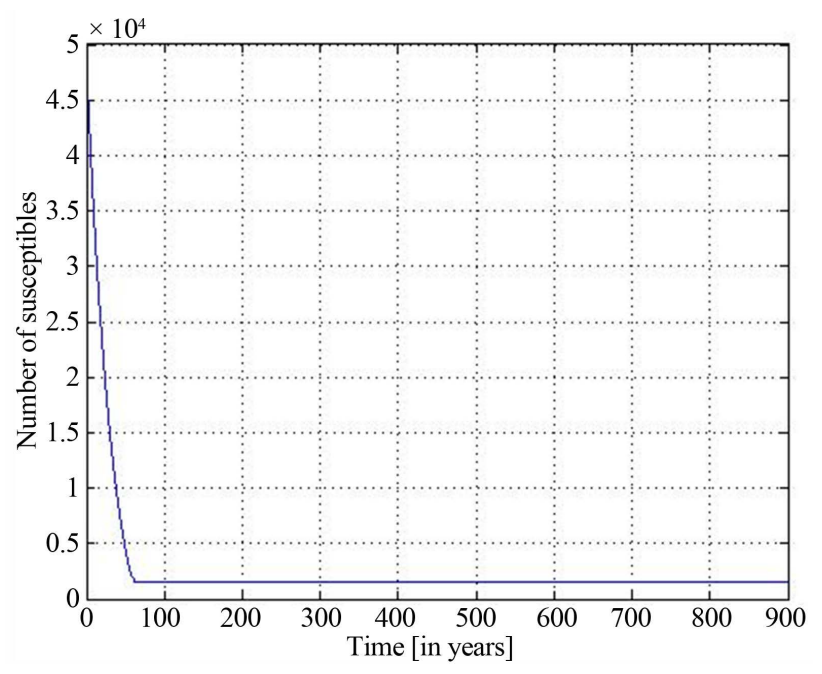

Figure 2. Function $t \mapsto \int_{0}^{A} S(t, a) d a$ with $R_{0}<1$.

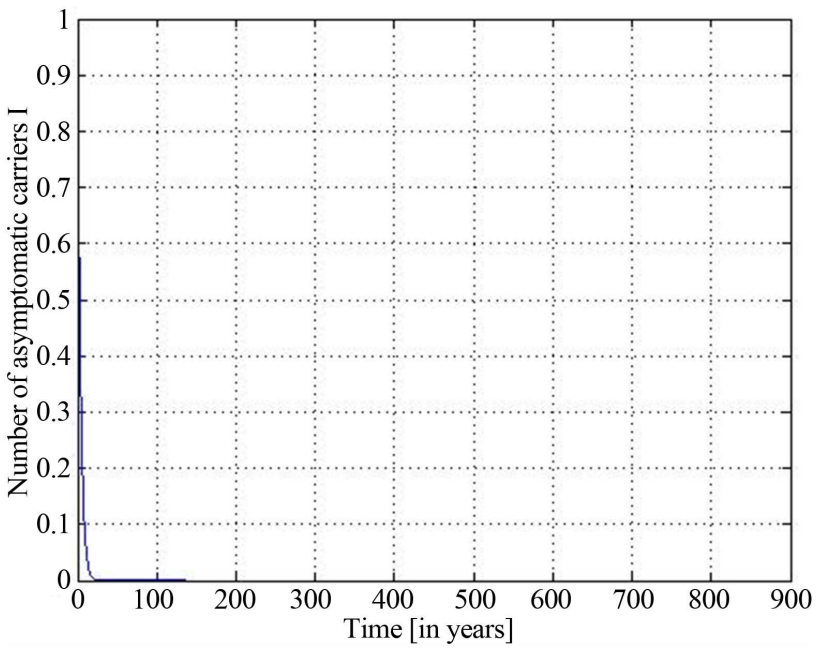

Figure 3. Function $I(t)$ with $R_{0}<1$.

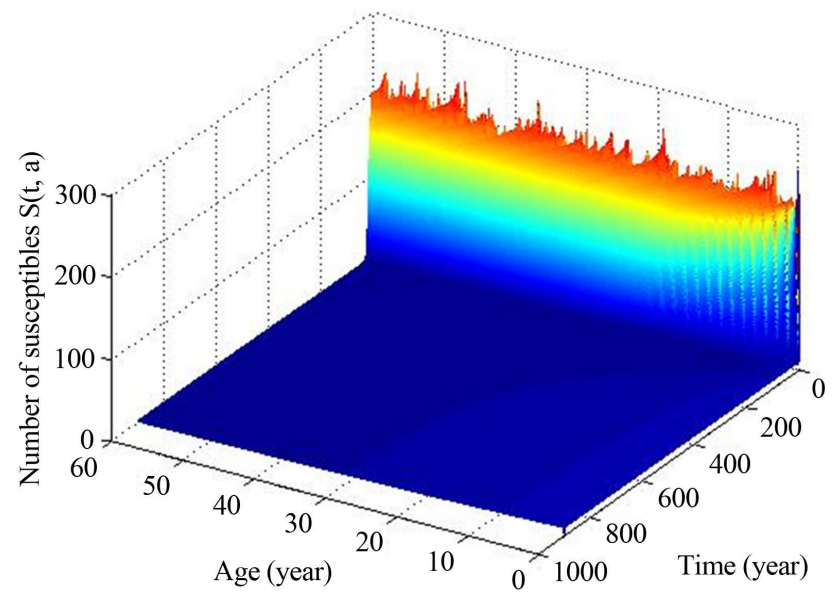

Figure 4. Function $S(t, a)$ with $R_{0}>1$. 


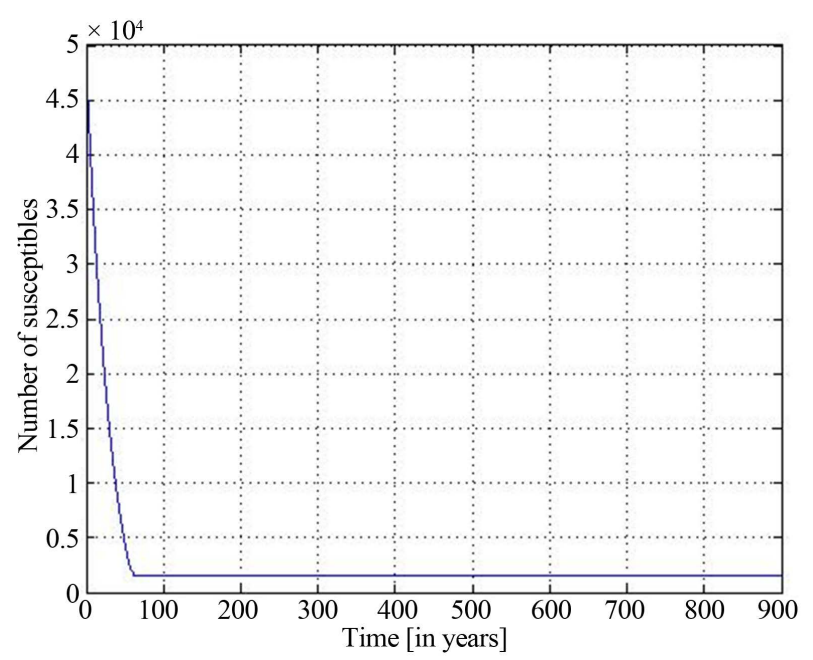

Figure 5. Function $t \mapsto \int_{0}^{A} S(t, a) d a$ with $R_{0}>1$.

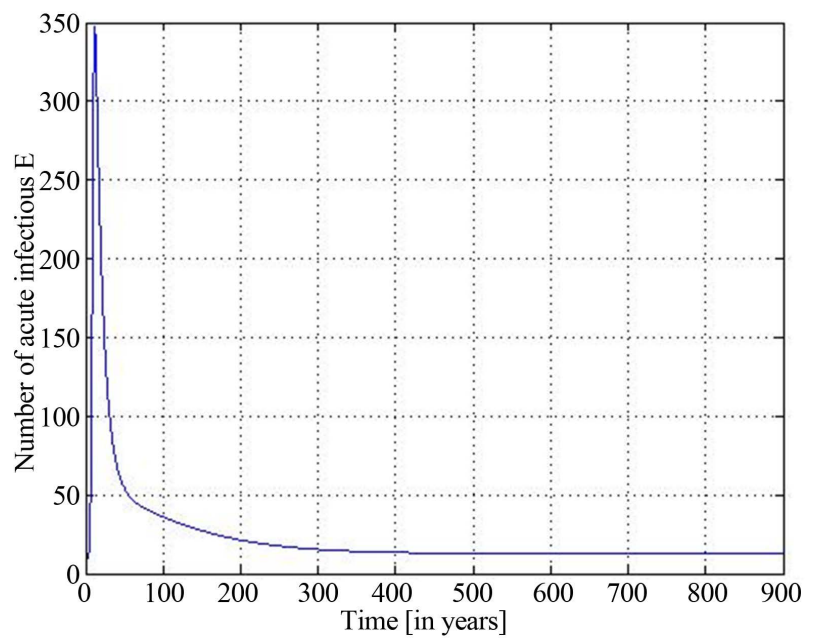

Figure 6. Function $E(t)$ with $R_{0}>1$.

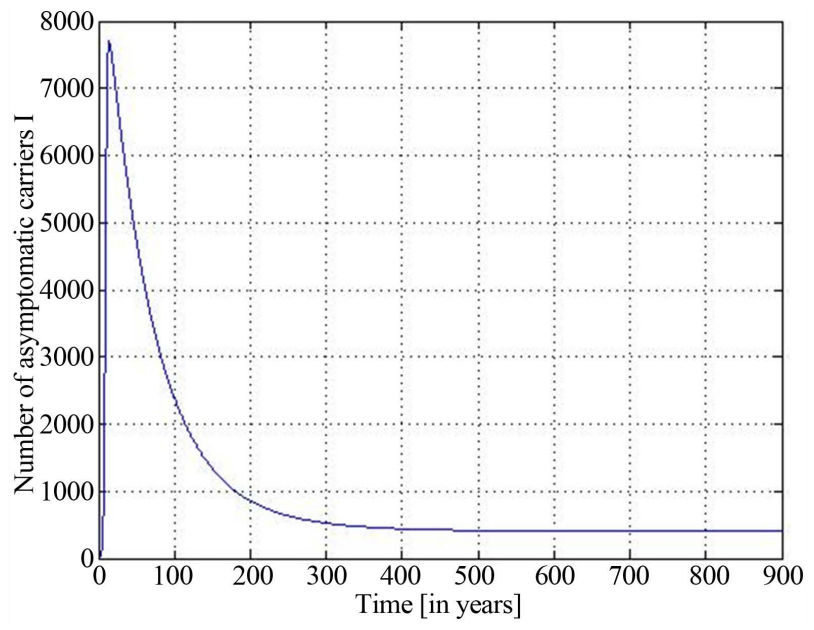

Figure 7. Function $I(t)$ with $R_{0}>1$. 


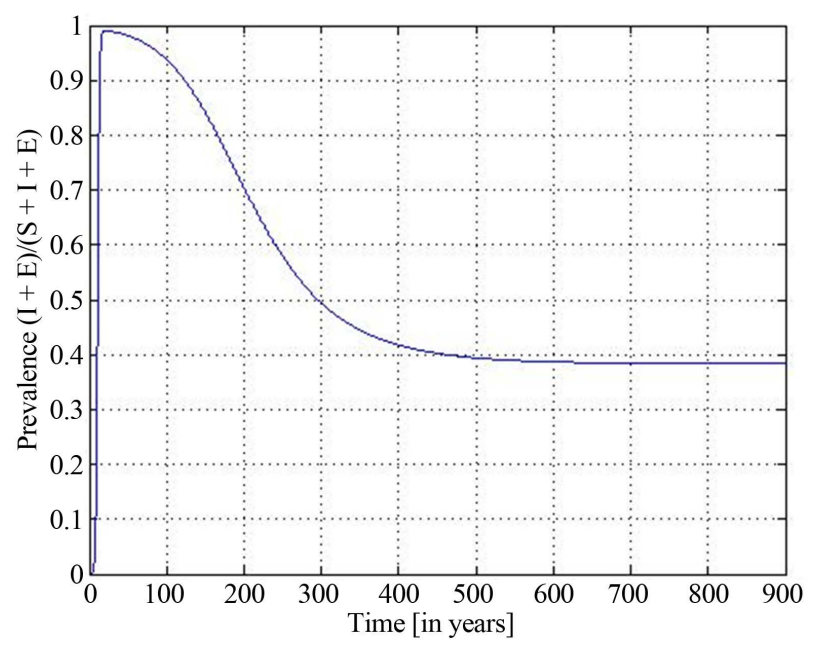

Figure 8. Function prevalence with $R_{0}>1$.

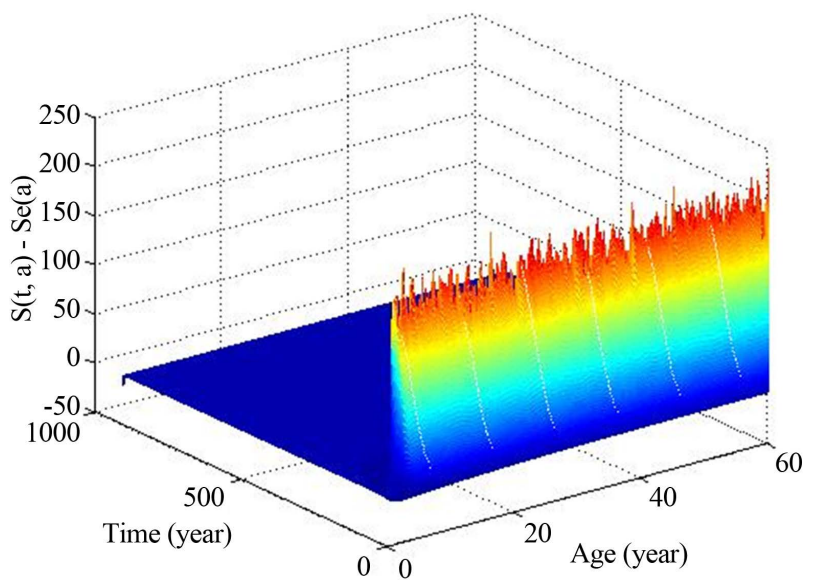

Figure 9. Positivity of $S(t, a)-S_{e}(a)$ with $R_{0}>1$.

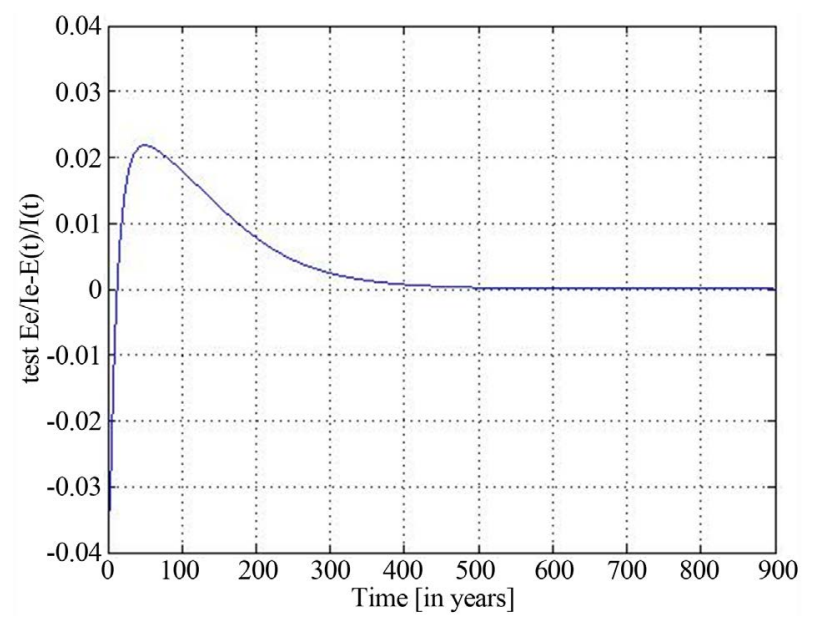

Figure 10. Positivity for long term dynamics of $\frac{E_{e}}{I_{e}}-\frac{E(t)}{I(t)}$ with $R_{0}>1$. 


$$
X_{+}=L^{1}(] 0 ;+\infty\left[, \mathbb{R}_{+}\right) \times \mathbb{R}_{+} \times \mathbb{R}_{+} \times \mathbb{R}_{+}
$$

with $X_{0+}=X_{0} \cap X_{+}$.

We consider also the linear operator $A: D(A) \subset X \rightarrow X$ defined by

$$
A\left(\begin{array}{c}
\varphi \\
0 \\
\alpha_{E} \\
\alpha_{I}
\end{array}\right)=\left(\begin{array}{c}
-\varphi^{\prime}-m(.) \varphi \\
-\varphi(0) \\
-\mu_{E} \alpha_{E} \\
-\mu_{I} \alpha_{I}
\end{array}\right)
$$

with the non densily domain $\left.\left.D(A)=W^{1,1}(] 0 ;+\infty\right)\right) \times\{0\} \times \mathbb{R} \times \mathbb{R}$ in $X: \overline{D(A)}=X_{0} \neq X$.

Finally let us introduce the nonlinear and Frechet differentiable map $F: \overline{D(A)} \rightarrow X$ defined by:

$$
F\left(\begin{array}{c}
\varphi \\
0 \\
\alpha_{E} \\
\alpha_{I}
\end{array}\right)=\left(\begin{array}{c}
-\beta_{I}(.) \alpha_{I} \varphi \\
\Lambda \\
\int_{0}^{+\infty} p(a) \beta_{I}(a) \alpha_{I} \varphi(a) \mathrm{d} a \\
\int_{0}^{+\infty} q(a) \beta_{I}(a) \alpha_{I} \varphi(a) \mathrm{d} a+\varepsilon \alpha_{E}
\end{array}\right)
$$

Identifying $(S(t,),. E(t), I(t))$ and $u(t)=(S(t, .), 0, E(t), I(t))^{\mathrm{T}}$, one obtains that System (1.1)-(1.5) rewrites as the following non-densely defined Cauchy problem (1.10):

$$
\begin{gathered}
\frac{\mathrm{d} u(t)}{\mathrm{d} t}=A u(t)+F(u(t)), t>0 \\
u(0)=\left(S_{0}(.), 0, E_{0}, I_{0}\right) \in X_{0+}
\end{gathered}
$$

We first derive that the above abstract Cauchy problem (1.10)-(1.11) generates a unique globally defined and positive semiflow. Moreover A satisfies the Hille-Yosida property. Then standard methodologies apply to provide the existence and uniqueness of mild solution for system (1.10)-(1.11) (see for instance [10] [14]-[17]):

Proposition 1. Let Mathematical Assumption 1 be satisfied.

Then there exists a continuous semiflow that is bounded dissipative $\{U(t)\}_{\text {t>0 }}$ on $X_{0+}$ into itself such that for each $x \in X_{0+}$, the map $t \rightarrow U(t) x$ is the unique integrated solution of (1.10)-(1.11) with initial data $x$, namely $t \rightarrow U(t) x$ satisfies

(i) $\int_{0}^{t} U(s) x \mathrm{~d} s \in D(A), \forall t \geq 0$,

(ii) $U(t) x=x+A \int_{0}^{t} U(s) x \mathrm{~d} s+\int_{0}^{t} F(U(s) x) \mathrm{d} s$ for each $t \geq 0$.

Remark 2. One can prove the proposition 1 by using ideas of corollaries 1 and 2 in Melnik et al. [11].

By using results in Sell and You [18], one can prove that $\{U(t)\}_{t \geq 0}$ is asymptotically smooth. Then using results of Hale [19] [20], Hale et al. [21], one obtains the following proposition.

Proposition 2. Let Mathematical Assumption 1 be satisfied. Then there exists a compact set $\mathcal{A} \subset X_{0+}$ such that

(i) $\mathcal{A}$ is invariant under the semiflow $\{U(t)\}_{t \geq 0}$.

(ii) $\mathcal{A}$ attracts the bounded sets of $X_{0+}$ under $\{U(t)\}_{t \geq 0}$. This means that for each bounded set $B \subset X_{0+}$ we have

$$
\lim _{t \rightarrow \infty} \delta(U(t) B, \mathcal{A})=0
$$

where $\delta$ is defined as

$$
\delta(A, B)=\operatorname{supinf}_{x \in A}\|x-y\| .
$$

Moreover $\mathcal{A}$ is locally asymptotically stable.

We will widely adapt ideas of Magal et al. [13] and Melnik et al. [11] here with Lyapunov functionals on $\mathcal{A}$ for the global stability of DFE and EE. 
1) Stability of the DFE: $R_{0}<1$

Let us introduce the positive map defined on $\mathbb{R}$ :

$$
V(t)=\int_{0}^{+\infty} A(a) G\left(\frac{S(t, a)}{S_{F}(a)}\right) \mathrm{d} a+B E(t)+C I(t) .
$$

is positive defin ite at the DFE. We evaluate $\frac{\mathrm{d} V(t)}{\mathrm{d} t}$ as

$$
\int_{0}^{+\infty} S_{F}(a) G\left(\frac{1}{S_{F}(a)}-\frac{1}{S(t, a)}\right) \frac{\partial S(t, a)}{\partial t} \mathrm{~d} a+\frac{\varepsilon}{\varepsilon p+\mu_{E} q} \frac{\mathrm{d} E(t)}{\mathrm{d} t}+\frac{\mu_{E}}{\varepsilon p+\mu_{E} q} \frac{\mathrm{d} I(t)}{\mathrm{d} t}
$$

with $A(a)=\left(q(a)+\frac{\mu_{E}}{\varepsilon} p(a)\right) S_{F}(a), B=1$ and $C=\frac{\mu_{E}}{\varepsilon}$. The equations of the system 1.1 help us to get for $\frac{\mathrm{d} V(t)}{\mathrm{d} t}$ :

$$
\frac{\mathrm{d} V(t)}{\mathrm{d} t}=-\int_{0}^{+\infty}\left(q(a)+\frac{\mu_{E}}{\varepsilon} p(a)\right) S_{F}(a)\left(\frac{S(t, a)}{S_{F}(a)}-1\right)\left(\frac{\partial_{a} S(t, a)}{S(t, a)}+m(a)\right) \mathrm{d} a+\frac{\mu_{E}}{\varepsilon} \mu_{I}\left(R_{0}-1\right) I(t)
$$

We would like to prove that

$$
\int_{0}^{+\infty}\left(q(a)+\frac{\mu_{E}}{\varepsilon} p(a)\right) S_{F}(a)\left(\frac{S(t, a)}{S_{F}(a)}-1\right)\left(\frac{\partial_{a} S(t, a)}{S(t, a)}+m(a)\right) \mathrm{d} a \geq 0 .
$$

Three cases occur by Assumption 3:

1. If $\left(\frac{S(t, a)}{S_{F}(a)}-1\right)\left(\frac{\partial_{a} S(t, a)}{S(t, a)}+m(a)\right)>0$, one obtains:

$$
\begin{aligned}
S_{F}(a)\left(\frac{S(t, a)}{S_{F}(a)}-1\right)\left(\frac{\partial_{a} S(t, a)}{S(t, a)}+m(a)\right) & <\left(q(a)+\frac{\mu_{E}}{\varepsilon} p(a)\right) S_{F}(a)\left(\frac{S(t, a)}{S_{F}(a)}-1\right)\left(\frac{\partial_{a} S(t, a)}{S(t, a)}+m(a)\right) \\
& <\frac{\mu_{E}}{\varepsilon} S_{F}(a)\left(\frac{S(t, a)}{S_{F}(a)}-1\right)\left(\frac{\partial_{a} S(t, a)}{S(t, a)}+m(a)\right)
\end{aligned}
$$

And by integrating from 0 to $+\infty$, one gets:

$$
\begin{aligned}
& \int_{0}^{+\infty} S_{F}(a)\left(\frac{S(t, a)}{S_{F}(a)}-1\right)\left(\frac{\partial_{a} S(t, a)}{S(t, a)}+m(a)\right) \mathrm{d} a \\
& \leq \int_{0}^{+\infty}\left(q(a)+\frac{\mu_{E}}{\varepsilon} p(a)\right) S_{F}(a)\left(\frac{S(t, a)}{S_{F}(a)}-1\right)\left(\frac{\partial_{a} S(t, a)}{S(t, a)}+m(a)\right) \mathrm{d} a \\
& \leq \int_{0}^{+\infty} \frac{\mu_{E}}{\varepsilon} S_{F}(a)\left(\frac{S(t, a)}{S_{F}(a)}-1\right)\left(\frac{\partial_{a} S(t, a)}{S(t, a)}+m(a)\right) \mathrm{d} a
\end{aligned}
$$

Then

$$
\int_{0}^{+\infty}\left(q(a)+\frac{\mu_{E}}{\varepsilon} p(a)\right) S_{F}(a)\left(\frac{S(t, a)}{S_{F}(a)}-1\right)\left(\frac{\partial_{a} S(t, a)}{S(t, a)}+m(a)\right) \mathrm{d} a>0 .
$$

2. If $\left(\frac{S(t, a)}{S_{F}(a)}-1\right)\left(\frac{\partial_{a} S(t, a)}{S(t, a)}+m(a)\right)=0$, one gets:

$$
\int_{0}^{+\infty}\left(q(a)+\frac{\mu_{E}}{\varepsilon} p(a)\right) S_{F}(a)\left(\frac{S(t, a)}{S_{F}(a)}-1\right)\left(\frac{\partial_{a} S(t, a)}{S(t, a)}+m(a)\right) \mathrm{d} a=0 .
$$


3. If $\left(\frac{S(t, a)}{S_{F}(a)}-1\right)\left(\frac{\partial_{a} S(t, a)}{S(t, a)}+m(a)\right)<0$, one gets:

$$
\begin{aligned}
\frac{\mu_{E}}{\varepsilon} S_{F}(a)\left(\frac{S(t, a)}{S_{F}(a)}-1\right)\left(\frac{\partial_{a} S(t, a)}{S(t, a)}+m(a)\right) & <\left(q(a)+\frac{\mu_{E}}{\varepsilon} p(a)\right) S_{F}(a)\left(\frac{S(t, a)}{S_{F}(a)}-1\right)\left(\frac{\partial_{a} S(t, a)}{S(t, a)}+m(a)\right) \\
& <S_{F}(a)\left(\frac{S(t, a)}{S_{F}(a)}-1\right)\left(\frac{\partial_{a} S(t, a)}{S(t, a)}+m(a)\right)
\end{aligned}
$$

And by integrating from 0 to $+\infty$, one gets:

$$
\begin{aligned}
& \frac{\mu_{E}}{\varepsilon} \int_{0}^{+\infty} S_{F}(a)\left(\frac{S(t, a)}{S_{F}(a)}-1\right)\left(\frac{\partial_{a} S(t, a)}{S(t, a)}+m(a)\right) \mathrm{d} a \\
& \leq \int_{0}^{+\infty}\left(q(a)+\frac{\mu_{E}}{\varepsilon} p(a)\right) S_{F}(a)\left(\frac{S(t, a)}{S_{F}(a)}-1\right)\left(\frac{\partial_{a} S(t, a)}{S(t, a)}+m(a)\right) \mathrm{d} a \\
& \leq \int_{0}^{+\infty} S_{F}(a)\left(\frac{S(t, a)}{S_{F}(a)}-1\right)\left(\frac{\partial_{a} S(t, a)}{S(t, a)}+m(a)\right) \mathrm{d} a .
\end{aligned}
$$

but $\frac{\mu_{E}}{\varepsilon} \geq 1$ and,

$$
\int_{0}^{+\infty} S_{F}(a)\left(\frac{S(t, a)}{S_{F}(a)}-1\right)\left(\frac{\partial_{a} S(t, a)}{S(t, a)}+m(a)\right) \mathrm{d} a \geq 0
$$

that implies

$$
\int_{0}^{+\infty}\left(q(a)+\frac{\mu_{E}}{\varepsilon} p(a)\right) S_{F}(a)\left(\frac{S(t, a)}{S_{F}(a)}-1\right)\left(\frac{\partial_{a} S(t, a)}{S(t, a)}+m(a)\right) \mathrm{d} a \geq 0 .
$$

Hence

$$
\int_{0}^{+\infty}\left(q(a)+\frac{\mu_{E}}{\varepsilon} p(a)\right) S_{F}(a)\left(\frac{S(t, a)}{S_{F}(a)}-1\right)\left(\frac{\partial_{a} S(t, a)}{S(t, a)}+m(a)\right) \mathrm{d} a \geq 0
$$

Finally

$$
\frac{\mathrm{d} V(t)}{\mathrm{d} t} \leq \frac{\mu_{E}}{\varepsilon} \mu_{I}\left(R_{0}-1\right) I(t) .
$$

Hence by recalling that $R_{0}<1$,

$$
\frac{\mathrm{d} V(t)}{\mathrm{d} t} \leq 0
$$

Finally by global stability Lyapunov-LaSalle theorem [11] [13] [22], the DFE $=\left(S_{F}(a), 0,0\right)$ is globally asymptotically stable because the largest invariant set of orbits $(S(t, a), I(t), E(t))$ verifying $\frac{\mathrm{d} V(t)}{\mathrm{d} t}=0$ is reduced for all positive $t$ and $a$, to $S(t, a)=S_{F}(a), I(t)=0$ and $E(t)=0$ corresponding to the disease free steady state (DFE) seen as $\left(S_{F}(a), 0,0\right)$.

2) Stability of the endemic equilibrium: $R_{0}>1$

Any solution of system (1.1) with positive initial condition remains positive indefinitely: then the system (1.1) is uniformly persistent (the tools are similar to Melnik [11]).

Let $G(x)=x-\ln x-1$. The function $G$ has only one extremum which is a global minimum 0 at 1 , satisfying $G(1)=0$ (see [13]). Then, we will analyse the Lyapunov functional

$$
V(t)=\int_{0}^{+\infty} A(a) G\left(\frac{S(t, a)}{S_{e}(a)}\right) \mathrm{d} a+B G\left(\frac{E(t)}{E_{e}}\right)+C G\left(\frac{I(t)}{I_{e}}\right) .
$$


We notice that $V\left(S_{e}(a), E_{e}, I_{e}\right)=0$ and $V$ is positive definite at $\mathrm{EE}=\left(S_{e}(a), E_{e}, I_{e}\right)$ that provides the minimum of $V$. Moreover $V$ is defined for all $S>0, E>0, I>0$ and

$$
\frac{\mathrm{d} V(t)}{\mathrm{d} t}=\int_{0}^{+\infty} A(a)\left(\frac{1}{S_{e}(a)}-\frac{1}{S(t, a)}\right) \frac{\partial S(t, a)}{\partial t} \mathrm{~d} a+B\left(\frac{1}{E_{e}}-\frac{1}{E(t)}\right) \frac{\mathrm{d} E(t)}{\mathrm{d} t}+C\left(\frac{1}{I_{e}}-\frac{1}{I(t)}\right) \frac{\mathrm{d} I(t)}{\mathrm{d} t},
$$

With

$$
B=E_{e}, C=\frac{\mu_{E}}{\varepsilon} I_{e} \text { and } A(a)=\left(q(a)+\frac{\mu_{E}}{\varepsilon} p(a)\right) S_{e}(a)
$$

we obtain:

$$
\begin{aligned}
\frac{\mathrm{d} V(t)}{\mathrm{d} t}= & -\int_{0}^{+\infty}\left(q(a)+\frac{\mu_{E}}{\varepsilon} p(a)\right) S_{e}(a)\left(\frac{S(t, a)}{S_{e}(a)}-1\right)\left(\frac{\partial_{a} S(t, a)}{S(t, a)}+m(a)+\beta_{I}(a) I_{e}\right) \mathrm{d} a \\
& +\int_{0}^{+\infty} q(a) S_{e}(a) G\left(\frac{S(t, a)}{S_{e}(a)}\right) \beta_{I}(a) I_{e} \mathrm{~d} a-\int_{0}^{+\infty} q(a) \beta_{I}(a) I_{e} S_{e}(a) G\left(\frac{I_{e} E(t)}{E_{e} I(t)}\right) \mathrm{d} a \\
& -\int_{0}^{+\infty} q(a) \beta_{I}(a) I_{e} S_{e}(a) G\left(\frac{I(t) E_{e} S(t, a)}{I_{e} E(t) S_{e}(a)}\right) \mathrm{d} a
\end{aligned}
$$

We set $H(t, a)=S_{e}(a)\left(\frac{S(t, a)}{S_{e}(a)}-1\right)\left(\frac{\partial_{a} S(t, a)}{S(t, a)}+m(a)+\beta_{I}(a) I_{e}\right)$. By assumption,

$$
p(a)+q(a)=1,0 \leq p(a) \leq 1 \text { and } 0<\varepsilon \leq \mu_{E} .
$$

Then

$$
1 \leq q(a)+\frac{\mu_{E}}{\varepsilon} p(a) \leq \frac{\mu_{E}}{\varepsilon} .
$$

Three cases appear by Assumption 2-1:

1. If $H(t, a)>0$, then

$$
H(t, a) \leq\left(q(a)+\frac{\mu_{E}}{\varepsilon} p(a)\right) H(t, a) \leq \frac{\mu_{E}}{\varepsilon} H(t, a) .
$$

By integrating from 0 to $+\infty$, one gets:

$$
\int_{0}^{+\infty}\left(q(a)+\frac{\mu_{E}}{\varepsilon} p(a)\right) S_{e}(a)\left(\frac{S(t, a)}{S_{e}(a)}-1\right)\left(\frac{\partial_{a} S(t, a)}{S(t, a)}+m(a)+\beta_{I}(a) I_{e}\right) \mathrm{d} a \geq \int_{0}^{+\infty} H(t, a) \mathrm{d} a .
$$

Then

$$
\begin{aligned}
\frac{\mathrm{d} V(t)}{\mathrm{d} t} \leq & -\left[S_{e}(a) G\left(\frac{S(t, a)}{S_{e}(a)}\right)\right]_{a=+\infty}-\int_{0}^{+\infty} m(a) S_{e}(a) G\left(\frac{S(t, a)}{S_{e}(a)}\right) \mathrm{d} a \\
& -\int_{0}^{+\infty} p(a) \beta_{I}(a) I_{e} S_{e}(a) G\left(\frac{S(t, a)}{S_{e}(a)}\right) \mathrm{d} a-\int_{0}^{+\infty} q(a) \beta_{I}(a) I_{e} S_{e}(a) G\left(\frac{I_{e} E(t)}{E_{e} I(t)}\right) \mathrm{d} a \\
& -\int_{0}^{+\infty} q(a) \beta_{I}(a) I_{e} S_{e}(a) G\left(\frac{I(t) E_{e} S(t, a)}{I_{e} E(t) S_{e}(a)}\right) \mathrm{d} a
\end{aligned}
$$

And finally $\frac{\mathrm{d} V(t)}{\mathrm{d} t} \leq 0$.

2. If $H(t, a)=0$, we get: $S(t, a)=S_{e}(a)$. But $\partial_{a} S(t, a)=-m(a) S(t, a)-\beta_{I}(a) I_{e} S(t, a)$.

1) For $S(t, a)=S_{e}(a)$ and $G(1)=0$, one has: 


$$
\int_{0}^{+\infty} q(a) S_{e}(a) G\left(\frac{S(t, a)}{S_{e}(a)}\right) \beta_{I}(a) I_{e} \mathrm{~d} a=0
$$

then

$$
\frac{\mathrm{d} V(t)}{\mathrm{d} t}=-\int_{0}^{+\infty} q(a) \beta_{I}(a) I_{e} S_{e}(a) G\left(\frac{I_{e} E(t)}{E_{e} I(t)}\right) \mathrm{d} a-\int_{0}^{+\infty} q(a) \beta_{I}(a) I_{e} S_{e}(a) G\left(\frac{I(t) E_{e} S(t, a)}{I_{e} E(t) S_{e}(a)}\right) \mathrm{d} a \leq 0 .
$$

2) For $\partial_{a} S(t, a)=-m(a) S(t, a)-\beta_{I}(a) I_{e} S(t, a)$, one obtains:

$$
\begin{aligned}
\frac{\mathrm{d} V(t)}{\mathrm{d} t}=\int_{0}^{+\infty} q(a) S_{e}(a) G\left(\frac{S(t, a)}{S_{e}(a)}\right) \beta_{I}(a) I_{e} \mathrm{~d} a & -\int_{0}^{+\infty} q(a) \beta_{I}(a) I_{e} S_{e}(a) G\left(\frac{I_{e} E(t)}{E_{e} I(t)}\right) \mathrm{d} a \\
& -\int_{0}^{+\infty} q(a) \beta_{I}(a) I_{e} S_{e}(a) G\left(\frac{I(t) E_{e} S(t, a)}{I_{e} E(t) S_{e}(a)}\right) \mathrm{d} a .
\end{aligned}
$$

To get $\frac{\mathrm{d} V(t)}{\mathrm{d} t} \leq 0$, it is enough to show that:

$$
G\left(\frac{I(t) E_{e} S(t, a)}{I_{e} E(t) S_{e}(a)}\right)+G\left(\frac{I_{e} E(t)}{E_{e} I(t)}\right)-G\left(\frac{S(t, a)}{S_{e}(a)}\right) \geq 0 .
$$

We set:

$$
x=\frac{I(t) E_{e}}{I_{e} E(t)} \text { and } y=\frac{S(t, a)}{S_{e}(a)},
$$

We want to prove that:

$$
G(x y)+G\left(\frac{1}{x}\right)-G(y) \geq 0 .
$$

By definition of $G(x)$ we have

$$
G(x)+G\left(\frac{1}{x}\right) \geq 0
$$

then

$$
G(x y)+G\left(\frac{1}{x}\right)-G(y) \geq G(x y)-G(x)-G(y) .
$$

It enough to verify this sufficient condition

$$
G(x y)-G(x)-G(y) \geq 0,
$$

Recall that

$$
G(x y)-G(x)-G(y)=(1-x)(1-y)
$$

Then

$$
G(x y)-G(x)-G(y) \geq 0
$$

if and only if

$$
1-x \geq 0 \text { and } 1-y \geq 0 \text { or } 1-x \leq 0 \text { and } 1-y \leq 0
$$

that means (see Figures 9 and 10 in the simulations of subsection 1.2.2)

$$
S(t, a) \geq S_{e}(a) \text { and } \frac{E(t)}{I(t)} \leq \frac{E_{e}}{I_{e}} \text { or } S(t, a) \leq S_{e}(a) \text { and } \frac{E(t)}{I(t)} \geq \frac{E_{e}}{I_{e}}
$$

and, 


$$
G(x y)-G(x)-G(y) \geq 0
$$

By Assumption 2-2,

$$
G\left(\frac{I(t) E_{e} S(t, a)}{I_{e} E(t) S_{e}(a)}\right)+G\left(\frac{I_{e} E(t)}{E_{e} I(t)}\right)-G\left(\frac{S(t, a)}{S_{e}(a)}\right) \geq 0
$$

we obtain,

$$
\begin{aligned}
& \int_{0}^{+\infty} p(a) S_{e}(a) G\left(\frac{S(t, a)}{S_{e}(a)}\right) \beta_{I}(a) I_{e} \mathrm{~d} a-\int_{0}^{+\infty} p(a) \beta_{I}(a) I_{e} S_{e}(a) G\left(\frac{I_{e} E(t)}{E_{e} I(t)}\right) \mathrm{d} a \\
& -\int_{0}^{+\infty} p(a) \beta_{I}(a) I_{e} S_{e}(a) G\left(\frac{I(t) E_{e} S(t, a)}{I_{e} E(t) S_{e}(a)}\right) \mathrm{d} a \leq 0
\end{aligned}
$$

and

$$
\frac{\mathrm{d} V(t)}{\mathrm{d} t} \leq 0
$$

3. If $H(t, a)<0$, then:

$$
\frac{\mu_{E}}{\varepsilon} H(t, a)<\left(q(a)+\frac{\mu_{E}}{\varepsilon} p(a)\right) H(t, a)<H(t, a) .
$$

By integrating from 0 to $+\infty$, one gets:

$$
\int_{0}^{+\infty}\left(q(a)+\frac{\mu_{E}}{\varepsilon} p(a)\right) S_{e}(a)\left(\frac{S(t, a)}{S_{e}(a)}-1\right)\left(\frac{\partial_{a} S(t, a)}{S(t, a)}+m(a)+\beta_{I}(a) I_{e}\right) \mathrm{d} a>\frac{\mu_{E}}{\varepsilon} \int_{0}^{+\infty} H(t, a) \mathrm{d} a .
$$

Then,

$$
\begin{aligned}
\frac{\mathrm{d} V(t)}{\mathrm{d} t}< & -\frac{\mu_{E}}{\varepsilon} \int_{0}^{+\infty} S_{e}(a)\left(\frac{S(t, a)}{S_{e}(a)}-1\right)\left(\frac{\partial_{a} S(t, a)}{S(t, a)}+m(a)+\beta_{I}(a) I_{e}\right) \mathrm{d} a \\
& +\int_{0}^{+\infty} q(a) S_{e}(a) G\left(\frac{S(t, a)}{S_{e}(a)}\right) \beta_{I}(a) I_{e} \mathrm{~d} a \\
& -\int_{0}^{+\infty} q(a) \beta_{I}(a) I_{e} S_{e}(a) G\left(\frac{I_{e} E(t)}{E_{e} I(t)}\right) \mathrm{d} a \\
& -\int_{0}^{+\infty} q(a) \beta_{I}(a) I_{e} S_{e}(a) G\left(\frac{I(t) E_{e} S(t, a)}{I_{e} E(t) S_{e}(a)}\right) \mathrm{d} a .
\end{aligned}
$$

but $\frac{\mu_{E}}{\varepsilon} \geq 1$. So

$$
\begin{aligned}
\frac{\mathrm{d} V(t)}{\mathrm{d} t} \leq & -\int_{0}^{+\infty} S_{e}(a)\left(\frac{S(t, a)}{S_{e}(a)}-1\right)\left(\frac{\partial_{a} S(t, a)}{S(t, a)}+m(a)+\beta_{I}(a) I_{e}\right) \mathrm{d} a \\
& +\int_{0}^{+\infty} q(a) S_{e}(a) G\left(\frac{S(t, a)}{S_{e}(a)}\right) \beta_{I}(a) I_{e} \mathrm{~d} a \\
& -\int_{0}^{+\infty} q(a) \beta_{I}(a) I_{e} S_{e}(a) G\left(\frac{I_{e} E(t)}{E_{e} I(t)}\right) \mathrm{d} a \\
& -\int_{0}^{+\infty} q(a) \beta_{I}(a) I_{e} S_{e}(a) G\left(\frac{I(t) E_{e} S(t, a)}{I_{e} E(t) S_{e}(a)}\right) \mathrm{d} a .
\end{aligned}
$$

by using results in case $H(t, a)>0$, one has: $\frac{\mathrm{d} V(t)}{\mathrm{d} t} \leq 0$. 
Then by global stability Lyapunov-LaSalle theorem [11] [13] [22], the endemic equilibrium (EE) is globally asymptotically stable because the largest invariant set of orbits $(S(t, a), I(t), E(t))$ verifying $\frac{\mathrm{d} V(t)}{\mathrm{d} t}=0$ is reduced for all positive $t$ and $a$, to $S(t, a)=S_{e}(a), I(t)=I_{e}$ and $E(t)=E_{e}$ corresponding to the endemic steady state $\left(S_{e}(a), E_{e}, I_{e}\right)$.

\section{Conclusions}

We observe that our computations for stability of DFE and EE are confirmed by simulations. It is also established that increasing the transmission coefficient $\beta_{I}$, increases the basic reproduction rate. In a forthcoming work, we will introduce vertical transmission (because of the contreversal article Sall et al. [23] on WHOs [12] neglection of vertical transmission in sub-Saharan Africa), studies of (optimal) vaccination strategies and immigration by other ways than birth. The results of this work extend those of Melnik et al. [11] and Kouakep et al. [10] on a more realistic case applied to hepatitis B situation. One can study the stability of the endemic equilibrium (EE) with $\beta_{E}$ small enough (like Ducrot et al. [10]) using perturbation arguments of Magal [24]. For the case (avoid here) where $\varepsilon=0$ and the map $a \mapsto \beta_{E}(a)$ is bounded and uniformly continuous from $[0, \infty)$ into itself, Ducrot et al. [25] deal with global stability of the disease free equilibrium with (constant) functions $\beta_{E}$ and $\beta_{I}$ by considering a particular case of the Lyapunov functional (similar to Magal et al. [13] and Kouakep et al. [10]) $V[I, E](t):=\int_{0}^{\infty}\left[\Gamma_{I}(\tau) I(t)+\Gamma_{E}(\tau) E(t)\right] \mathrm{d} \tau$ which is non-increasing along the complete orbits with $\Gamma_{I}$ and $\Gamma_{E}$ such that

$$
\left\{\begin{array}{l}
\Gamma_{I}^{\prime}(a)=\mu_{I} \Gamma_{I}(\tau)-\beta_{I}(a) \\
\Gamma_{I}(0)=\int_{0}^{\infty} \mathrm{e}^{-\mu_{I} s} \beta_{I}(s) \mathrm{d} s,
\end{array}\right.
$$

and

$$
\left\{\begin{array}{l}
\Gamma_{E}^{\prime}(a)=\mu_{E} \Gamma_{E}(\tau)-\beta_{E}(a) \\
\Gamma_{E}(0)=\int_{0}^{\infty} \mathrm{e}^{-\mu_{E} s} \beta_{E}(s) \mathrm{d} s,
\end{array}\right.
$$

Note that for $\varepsilon=0$,

$$
R_{0}=\Gamma_{I}(0) \int_{0}^{\infty} p(a) s_{F}(a) \mathrm{d} a+\Gamma_{E}(0) \int_{0}^{\infty}(1-p(a)) s_{F}(a) \mathrm{d} a .
$$

Ducrot et al. [25] used also arguments like those in (Demasse et al. [17], proposition 4.1 and its proof). For global stability of endemic equilibrium in the case $\varepsilon=0$, Ducrot et al. [25] used the following Lyapunov functional (under special assumptions on $p$ ) with a well-chosen positive constant $K$ :

$$
V[S, I](t)=\int_{0}^{\infty} p(a) S_{e}(a) G\left(\frac{S(t, a)}{S_{e}(a)}\right) \mathrm{d} a+K G\left(\frac{E(t)}{E_{e}}\right),
$$

The model (1.1)-(1.5) is formally equivalent (with $I(t):=\int_{0}^{\infty} i(t, a) \mathrm{d} a$ and $E(t):=\int_{0}^{\infty} e(t, a) \mathrm{d} a$ ) to the following model:

$$
\begin{aligned}
& {\left[\partial_{t}+m(a)\right] S(t, a)=-\lambda(t, a) S(t, a), t>0, a>0} \\
& {\left[\partial_{t}+\partial_{\tau}+\mu_{E}\right] e(t, a)=\lambda(t, a) p(a) S(t, a),} \\
& {\left[\partial_{t}+\partial_{\tau}+\mu_{I}\right] i(t, a)=\lambda(t, a) q(a) S(t, a)+\varepsilon e(t, a),} \\
& \lambda(t, a)=\left[\beta_{I}(a) i(t, a)+\beta_{E}(a) e(t, a)\right] .
\end{aligned}
$$

supplemented together with the boundary conditions:

$$
\begin{aligned}
& S(t, 0)=\Lambda,(\text { constant influx }), \\
& e(t, 0)=e(t, 0)=0,(\text { no vertical transmission }), \\
& i(t, 0)=0,(\text { no immunity at birth }),
\end{aligned}
$$


and initial data

$$
S(0, a)=S_{0}(a) .
$$

By replacing $a$ (chronological age) by $\tau$ (infection age) in the infectives classes $e$ and $i$, the model (1.1)-(1.5) is equivalent (with $\varepsilon=0$ ) to the following model (see Kouakep et al. [10]):

$$
\begin{aligned}
& \left(\partial_{t}+\partial_{a}\right) S(t, a)=-\mu S(t, a)-\lambda(t) S(t, a), t>0, a>0, \\
& S(t, 0)=\Lambda, \\
& \left(\partial_{t}+\partial_{\tau}\right) e(t, \tau)=-\left(\mu+\gamma_{e}\right) e(t, \tau), t>0, \tau>0, \\
& \left(\partial_{t}+\partial_{\tau}\right) i(t, \tau)=-\left(\mu+\gamma_{i}\right) i(t, \tau), t>0, \tau>0, \\
& e(t, 0)=\lambda(t) \int_{0}^{\infty} p(a) S(t, a) \mathrm{d} a, i(t, 0)=\lambda(t) \int_{0}^{\infty} q(a) S(t, a) \mathrm{d} a .
\end{aligned}
$$

supplemented together with the boundary conditions:

$$
\begin{aligned}
& S(t, 0)=\Lambda,(\text { constant influx }) \\
& S(0, .)=S_{0}(.) \in L_{+}^{1}(0, \infty) \\
& i(0, .)=i_{0}(.), e(0, .)=e_{0}(.) \text { with }\left(i_{0}, e_{0}\right) \in\left[L_{+}^{1}(0, \infty)\right]^{2} .
\end{aligned}
$$

it remains to model $\lambda(t, a)$, the force of infection, and those general form can be written in the form

$$
\lambda(t)=\int_{0}^{\infty}\left(\beta_{I}(\tau) i(t, \tau)+\beta_{E}(\tau) e(t, \tau)\right) \mathrm{d} \tau .
$$

where $a$ is the chronological age and $\tau$ is the time since the infective(s) are contaminated. Another similar problem (with $m(a) \equiv \mu \in[0 ;+\infty)$ ) is:

$$
\begin{aligned}
& {\left[\partial_{t}+\mu\right] s(t)=\Lambda-\lambda(t, a) s(t), t>0,} \\
& {\left[\partial_{t}+\partial_{\tau}+\mu_{E}\right] e(t, \tau)=\lambda(t) p(\tau) s(t), \tau>0} \\
& {\left[\partial_{t}+\partial_{\tau}+\mu_{I}\right] i(t, \tau)=\lambda(t) q(\tau) s(t)+\varepsilon e(t, \tau), \tau>0} \\
& \lambda(t)=\int_{0}^{\infty}\left[\beta_{I}(\tau) i(t, \tau)+\beta_{E}(\tau) e(t, \tau)\right] \mathrm{d} \tau .
\end{aligned}
$$

supplemented together with the boundary conditions:

$$
\begin{aligned}
& s(0)=s_{0} \in[0 ;+\infty), \\
& e(t, 0)=e(t, 0)=0,(\text { no vertical transmission }), \\
& i(t, 0)=0,(\text { no immunity at birth }),
\end{aligned}
$$

We strongly believe that Assumptions 2 and 3 could be relaxed if we use usual tools of functional analysis by splitting functions $H$ and $P$ in the form $F^{+}-F^{-}$as a difference of two well-chosen positive functions $F^{+}$and $F^{-}$. Then one can use the constant-sign cases on $F^{+}$and $F^{-}$.

\section{Acknowledgements}

Authors are grateful to Reviewers, Pr Bekolle, Dr. A. Ducrot, Dr. Damakoa Irepran, Dr. Kamgang J. C. and GDM-MIAP group for helpful remarks or comments on the manuscript.

\section{References}

[1] Houpa, D.D.E., Miamdjo Tagne, E. and Kouakep, T.Y. (2014) A Model for Hepatitis B Disease with Age-Dependent Susceptibility. JMCS, 4, accepted. http://scik.org/index.php/jmcs/article/view/1431

[2] Zuckerman, A.J. (1976) The A, B, C Viruses. Nature, 259, 363-364.

[3] Fall, A.A., Gauthier, S. and Abderrahman, I. (2010) Modélisation de la Transmission Verticale de l'Hépatite B. CARI 2010 Report. 
[4] WHO (2013) Centre des Médias: Hépatite B. http://www.who.int/mediacentre/factsheets/fs204/en/index.html

[5] Pasquini, P. and Cvjetanović, B. (1988) Mathematical Models of Hepatitis B Infection. Annali dell'Istituto Superiore di Sanità, 24, 245-250.

[6] Bonzi, B., Fall, A.A., Iggidr, A. and Sallet, G. (2011) Stability of Differential Susceptibility and Infectivity Epidemic Models. Journal of Mathematical Biology, 62, 39-64. http://dx.doi.org/10.1007/s00285-010-0327-y

[7] Inaba, H. (1990) Threshold and Stability Results for an Age-Structured Epidemic Model. Journal of Mathematical Biology, 28, 411-434. http://dx.doi.org/10.1007/BF00178326

[8] Nokes, D.J., Hall, A.J., Edmunds, W.J., Medley, G.F. and Whittle, H.C. (1993) The Influence of Age on the Development of the Hepatitis B carrier State. Proceedings of the Royal Society B: Biological Sciences, 253, 197-201. http://dx.doi.org/10.1098/rspb.1993.0102

[9] Zou, L., Ruan, S. and Zhang, W. (2010) An Age-Structured Model for Transmission Dynamics of Hepatitis B. SIAM, 70, 3121-3139.

[10] Kouakep, T.Y., Ducrot, A. and Houpa, D.D.E. (2013) A Model for Hepatitis B with Chronological and Infection Ages. Applied Mathematical Sciences, 7, 5977-5993.

[11] Melnik, A.V. and Korobeinikov, A. (2013) Lyapunov Functions and Global Stability for SIR and SEIR Models with Age-Dependent Susceptibility. Mathematical Biosciences and Engineering, 10, 369-378. http://dx.doi.org/10.3934/mbe.2013.10.369

[12] WHO (1996) Hepatitis B and Breastfeeding. http://www.who.int/maternal_child_adolescent/documents/pdfs/hepatitis_b_and_breastfeeding.pdf

[13] Magal, P., McCluskey, C.C. and Webb, G.F. (2010) Liapunov Functional and Global Asymptotic Stability for an Infection-Age Model. Applicable Analysis, 89, 1109-1140. http://dx.doi.org/10.1080/00036810903208122

[14] Magal, P. (2001) Compact Attractors for Time-Periodic Age Structured Population Models. Electronic Journal of Differential Equations, 2001, 1-35.

[15] Magal, P. and Ruan, S. (2009) On Semilinear Cauchy Problems with Non-Dense Domain. Advances in Differential Equations, 14, 1041-1084.

[16] Thieme, H.R. (1997) Quasi-Compact Semigroups via Bounded Pertubation. In: Arino, O., Axelrod, D. and Kimmel, M., Eds., Advances in Mathematical Population Dynamics-Molecules, Cells and Man, World Scientific Publishing, River Edge, 691-711.

[17] Djidjou, D.R. and Ducrot, A. (2013) An Age-Structured Within-Host Model for Multistrain Malaria Infections. SIAM Journal on Applied Mathematics, 73, 572-593. http://dx.doi.org/10.1137/120890351

[18] Sell, G.R. and You, Y. (2002) Dynamics of Evolutionary Equations. Springer, New York. http://dx.doi.org/10.1007/978-1-4757-5037-9

[19] Hale, J.K. (1986) Asymptotic Behavior and Dynamics in Infinite Dimensions, in Nonlinear Differential Equations. Hale, J.K. and Martinez-Amores, P., Eds., Pitman, Marshfield.

[20] Hale, J.K. (1988) Asymptotic Behavior of Dissipative Systems. Mathematical Surveys and Monographs 25, American Mathematical Society, Providence.

[21] Hale, J.K. and Waltman, P. (1989) Persistence in Infinite-Dimensional Systems. SIAM Journal on Mathematical Analysis, 20, 388-395. http://dx.doi.org/10.1137/0520025

[22] LaSalle, J.P. (1976) The Stability of Dynamical Systems. SIAM, Philadelphia. http://dx.doi.org/10.1137/1.9781611970432

[23] Sall Diallo, A., Sarr, M., Fall, Y., Diagne, C. and Kane, M.O. (2004) Hepatitis B Infection in Infantile Population of Senegal. Dakar Medical, 49, 136-142.

[24] Magal, P. (2009) Perturbation of a Globally Stable Steady State and Uniform Persistence. Journal of Dynamics and Differential Equations, 21, 1-20. http://dx.doi.org/10.1007/s10884-008-9127-0

[25] Ducrot, A., Houpa, D.D.E. and Kouakep, T.Y. (2013) An Age-Structured Model with Differential Infectiousness: Application to Hepatitis B Virus Transmission. 